\section{Ultrastructure of telocytes, a new type of interstitial cells in the myocardium of the Chinese giant salamander (Andrias davidianus)}

\author{
Tingting Ge, ${ }^{1,2}$ Yaqiong Ye, ${ }^{1}$ Hui Zhang ${ }^{1,2}$ \\ ${ }^{1}$ College of Life Science and \\ Engineering, Foshan University \\ ${ }^{2}$ College of Animal Science and \\ Technology, Jiangxi Agricultural \\ University, Nanchang, China
}

\begin{abstract}
Telocytes (TCs) are new interstitial cells, and they are involved in tissue regeneration, particularly in heart. Therefore, TCs are suggested to be promising cells in regenerative medicine. However, the information of location structural characteristics and functions of TCs is still limited. In this study, cardiac TCs of the Chinese giant salamanders (Andrias davidianus) were identified by transmission electron microscopy. TCs were located in the interstitium between cardiomyocytes (CM). TCs possessed distinctive ultrastructural characteristics, including one to two very long and thin moniliform telopodes (Tps), emerging points from the cell body, caveolae, dichotomous branchings, labyrinthic systems, neighbouring exosomes and homocellular contacts between Tps. TCs/Tps were frequently observed in close proximity to cardiomyocytes. Moreover, Tps established hetero-cellular contacts with cardiomyocytes. Our results confirm the presence of TCs in the myocardium of the $A$. davidianus. This will help us to better understand roles of TCs in amphibian hearts.
\end{abstract}

\section{Introduction}

Telocytes (TCs), formerly called interstitial Cajal-like cells (ICLC), are a novel type of interstitial cells and play various important roles, such as mechanical supporting, intercellular communication, regulating surrounding cell type functions and homeostasis maintenance in tissues. ${ }^{1,2}$ In particular, TCs play a key role in tissue renewal and repair in various organs. ${ }^{2-4}$ In addition, in pathology, TCs are involved in tissue hyperplasia, chronic inflammation, systemic sclerosis, primary Sjögren's syndrome and psoriasis. ${ }^{5,6}$ Both in physiological and pathological conditions, TCs are multifunctional interstitial cells in tissues.
TCs have been identified in various organs of many animals. These studies of TCs were frequently performed in the hearts of human and other mammalians, including rat, mouse and pig, since TCs are supposed to participate in the regulation of cardiac homeostasis and regeneration..$^{7,8}$ Moreover, a significant link exists between the quantity of TCs in tissue and numerous cardiovascular diseases. ${ }^{9}$ Cardiac TCs were identified in two lower vertebrates, zebrafish and newt. ${ }^{9,10}$ Currently, a study identified TCs in an invertebrate (leech). ${ }^{11}$ However, the knowledge of cardiac TCs of lower vertebrates are still limited. Herein, we attempted to identify cardiac TCs of an amphibian - Chinese giant salamanders (Andrias davidianus) - by transmission electron microscopy (TEM), and to disclose structural relationships between TCs and cardiomyocytes (CM) and get the insight of physiological functions of TCs in amphibian hearts.

\section{Materials and Methods}

\section{Animals and sample tissues}

This work was approved by the Ethical Committee for Animal Care and Use of Jiangxi Agricultural University after relevant ethical review according to the National Institutes of Health Guide for the Care and Use of Laboratory Animals. The cultured commercial Andrias davidianus raised with simulated ecological breeding technology in their primary habitat were generously provided by an artificial breeding farm in mountain area, Jiangxi Province, China. The $A$. davidianus were placed on ice to make them dormant and euthanized, and they were quickly executed by destruction of the spine. All measures were taken to minimize the suffering of the experimental animals. The myocardium samples were quickly excised and fixed in $4 \%$ formaldehyde or $2.5 \%$ glutaraldehyde/phosphate-buffered saline (PBS) $(\mathrm{pH} 7.4,0.1 \mathrm{M})$ at $4^{\circ} \mathrm{C}$, respectively.

\section{Histology}

The myocardium samples were sampled and fixed in $4 \%$ formaldehyde. The samples were dehydrated in ethanol with gradient concentration, and embedded in paraffin. The samples were cut on a microtome (Jinhua, China). After dewaxing by xylene, the sections were stained with hematoxylineosin (H\&E) or toluidine blue, respectively. The sections were dehydrated and mounted, and observed with a BM 2000 light microscopy (Nanjing, China). The photomicrographs were acquired by ScopeImage 9.0 (H3D) software (Nanjing, China).
Correspondence: Hui Zhang, College of Life Science and Engineering, Foshan University, Foshan 528231, China

E-mail: zhanghui429@hotmail.com

Key words: Amphibian; cardiomyocytes; telocytes; telopodes; ultrastructure.

Contribution: HZ, project conception, research design, TEM analysis, data interpretation and manuscript drafting; TG, YY, experiments performing.

Conflict of interest: The authors declare no conflict of interest.

Funding: This work was supported by the National Natural Science Foundation of China (No. 31560681 and 31760716), the Project of Jiangxi Province (No. 20151BBF60007 and 20171ACB21028) and the Project of Education Department of Jiangxi Province (No. GJJ160418).

Received for publication: 27 February 2019. Accepted for publication: 10 May 2019.

This work is licensed under a Creative Commons Attribution-NonCommercial 4.0 International License (CC BY-NC 4.0).

(C) Copyright: the Author(s), 2019

Licensee PAGEPress, Italy

European Journal of Histochemistry 2019; 63:3021 doi:10.4081/ejh.2019.3021

\section{Transmission electron microscopy}

The TEM protocol was conducted in accordance with our previous methods. ${ }^{12-14}$ The myocardia were sampled and fixed in $2.5 \%$ glutaraldehyde/PBS $(\mathrm{pH} 7.4,0.1 \mathrm{M})$ at $4{ }^{\circ} \mathrm{C}$ for $48 \mathrm{~h}$, and then they were postfixed in $1 \% \mathrm{OsO}_{4}$ (Polysciences Inc., Warrington, PA, USA) for $1 \mathrm{~h}$, dehydrated in a concentration series of ethanol, infiltrated with propylene oxide/Araldite mixture and embedded in Araldite. The samples were sectioned, and they were stained with $1 \%$ uranyl acetate and Reynold's lead citrate for $20 \mathrm{~min}$. The stained sections were observed and photographed by a Hitachi H7650 TEM with a high-resolution digital camera (Tokyo, Japan).

\section{Results}

\section{Structural features of TCs}

In photomicrographs, TCs appear as cone-, spindle- and polygon-shaped with 1 , 2 and 3 long cytoplasmic processes, telopodes (Tps) (Figure 1). TCs are located between cardiomyocytes. In addition, by TEM, the ultrastructures of TCs are 
observed. TCs exhibit calabash-shaped (Figure 2A) and cone-shaped (Figure 2B) with an irregular contour, and contained a big nucleus and a small amount of cytoplasm. TCs with two very long and slender Tps are located in the interstitium between myocardial cells (Figures 2-5). Tps of TCs contain short dichotomous branchings (Figure 2B) and form labyrinthic systems in the convolute segments (Figure 2C). The long Tps consist of thin podomers and thick podoms (Figures 3A and 4 A,B,D). The thick podoms contain caveolae and mitochondria (Figures 3A, 5D). In addition, the exosomes are found and clung to Tps (Figure 4C).

\section{Structure relationships between TCs/Tps and surrounding cardiomyocytes}

The TCs and their long Tps are observed in close proximity to cardiomyocytes, which contained many myofibrils (CM) (Figures 1-5). TCs surround frequently the cardiomyocytes closely. Tps establish hetero-cellular close contacts with $\mathrm{CM}$ (Figures 3B and 5B). Also, Tps establish homo-cellular close contacts with other Tps (Figures 2 A,B and 5 C,D). Interestingly,

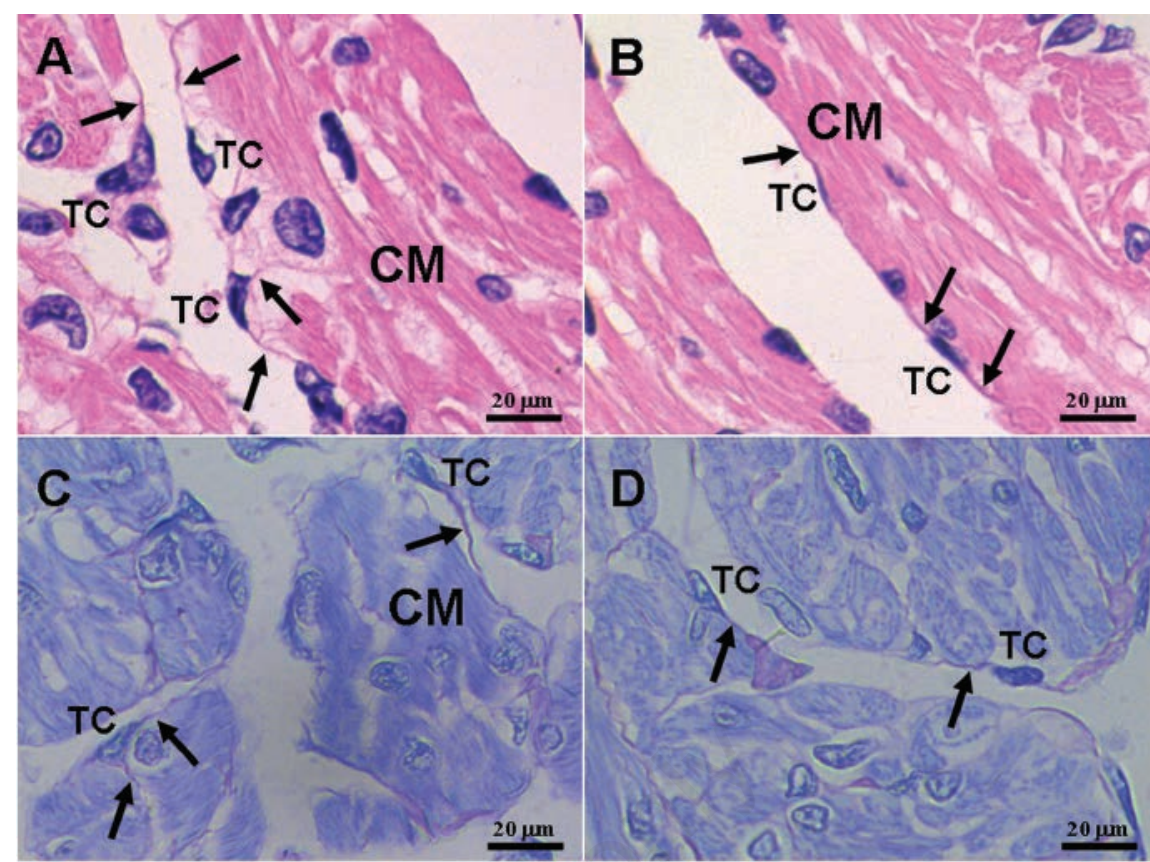

Figure 1. Light microphotographs of the myocardium of the A. davidianus. A,B) H\&E staining. C,D) Toluidine blue staining. Telocytes (TC) with long and thin telopodes (Tp; black arrows) are located between cardiomyocytes (CM), and TCs also surround cardiomyocytes (CM).

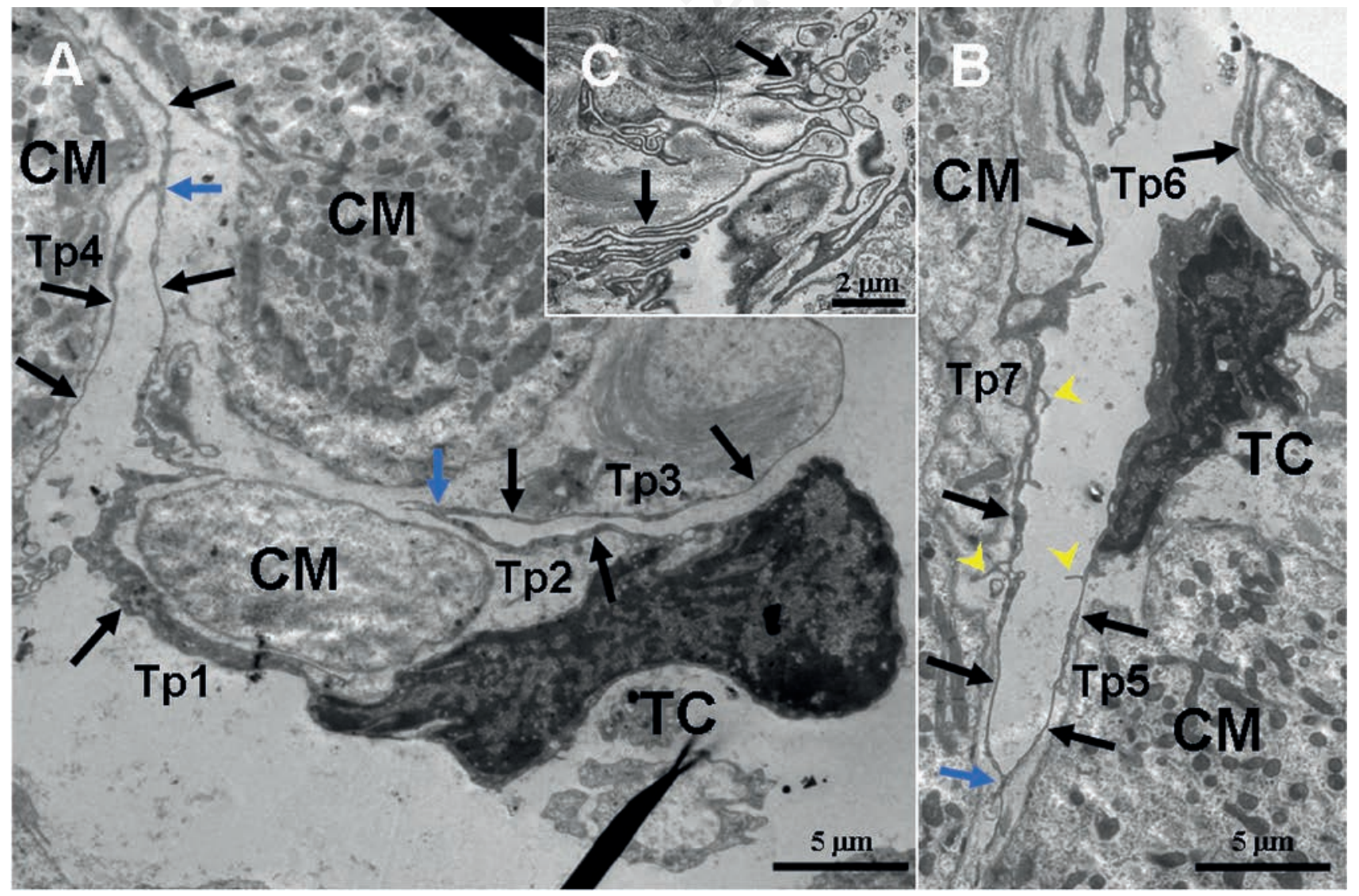

Figure 2. TEM micrographs of the myocardium of the A. davidianus. A,B) Two telocytes (TC) with long and thin telopodes (Tp; black arrows) including short branchings (yellow arrowheads,) are observed between cardiomyocytes (CM); TCs and their Tps are found close to CM; the homo-cellular close contacts (blue arrow) are established between Tps. C) Sinuous Tps form labyrinthic systems. 


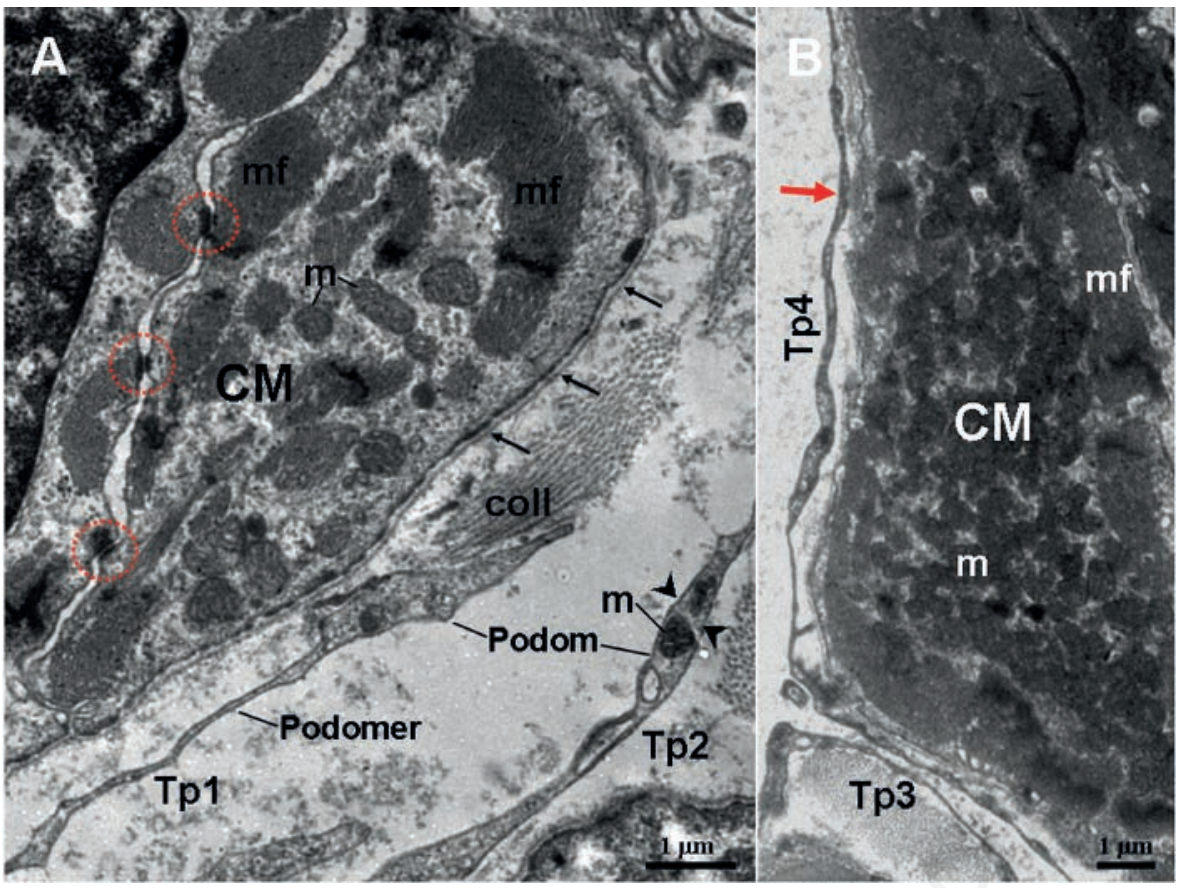

Figure 3. TEM micrographs of the myocardium of the A. davidianus. A) Two telopods ( $\mathrm{T} p 1$ and $\mathrm{Tp} 2)$ are observed in close proximity to cardiomyocyte $(\mathrm{CM})$, which contains mitochondria $(\mathrm{m})$ and myofibrils $(\mathrm{mf})$; abundant caveolae (arrowheads) are located near myofibrils. Tp consist of thin podomers and thick podoms. The podoms contain mitochondria and caveolae. B) Tp surrounds a CM; a close contact (red arrow) between $\mathrm{Tp} 4$ and $\mathrm{CM}$ is observed; black thin arrows note a continuous basal lamina; red outline notes desmosome. coll, collagen fibers.

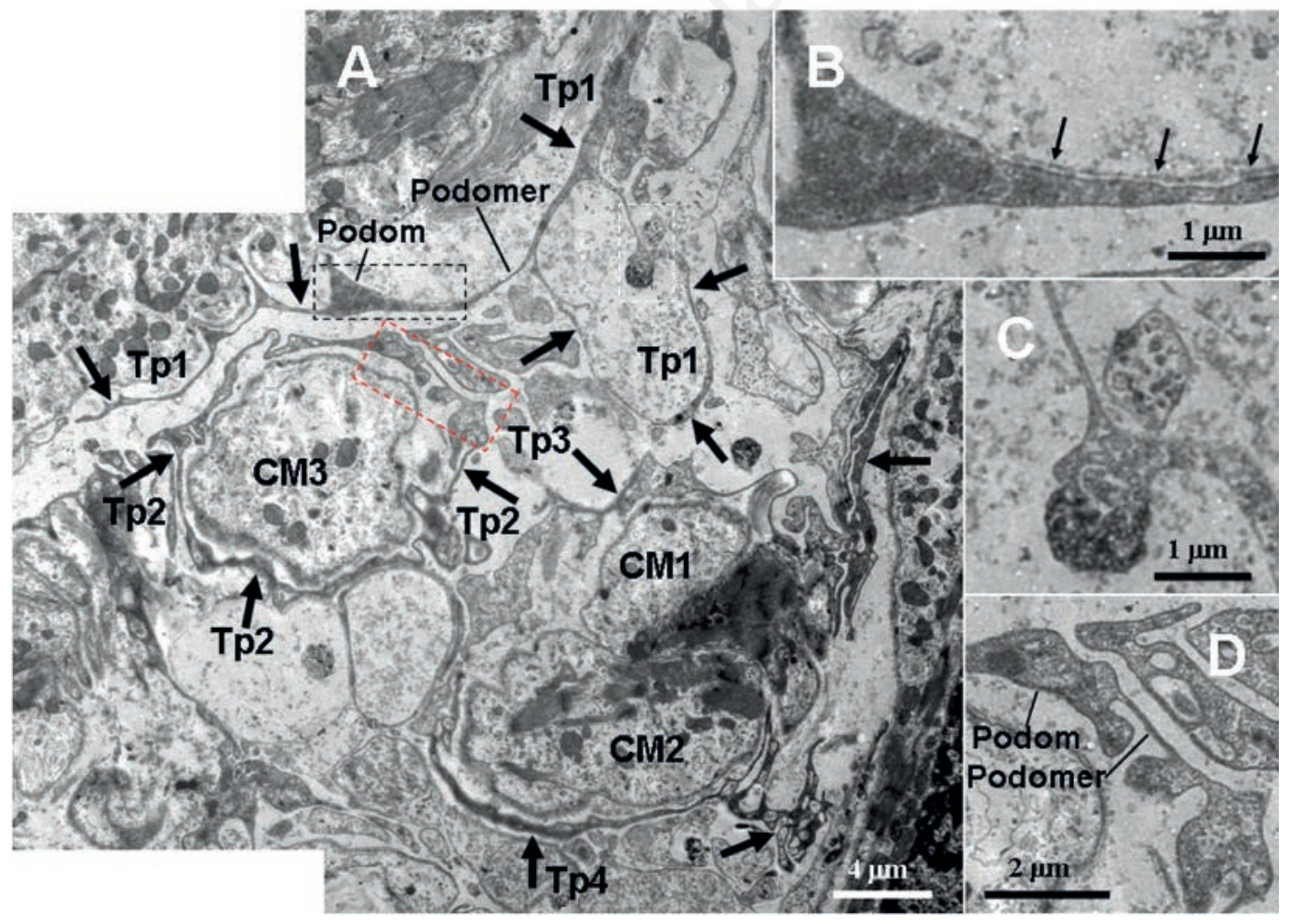

Figure 4. TEM micrographs of the myocardium of the $A$. davidianus. A) Two telopods (Tp3 and Tp4, black thick arrows) form a nonclosed ring and surround two cardiomyocytes (CM1 and CM2); the closed Tp2 surrounds a cardiomyocyte (CM3); the Tp1 contains podoms and podomers, and its segments form a closed loop; two exosomes (white dashed line boxed area) are found in close proximity to the Tp1 loop. B) High-magnification TEM micrograph of the black dashed line boxed areas shown in (A) with details of Tps segment containing a non-continuous basal lamina (black thin arrows). C) High-magnification TEM micrograph of the white dashed line boxed areas shown in (A) with details of the exosomes. D) High-magnification TEM micrograph of the red dashed line boxed areas shown in (A) with details of the Tp segments, which contains thick podom and thin podomer. 


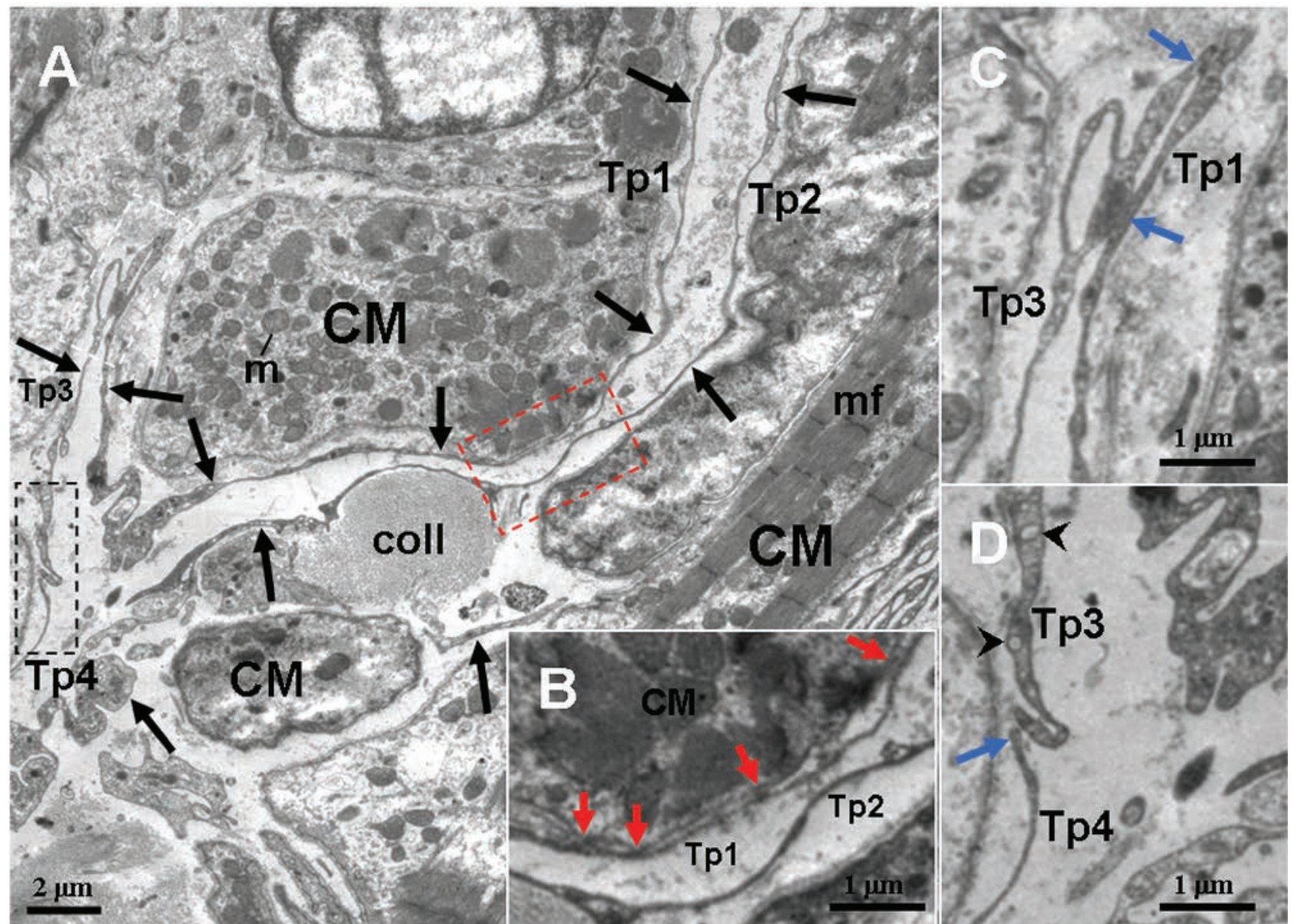

Figure 5. TEM micrographs of the myocardium of the $A$. davidianus. A) Four telopods (Tp1-4, black arrows) are observed in vicinity of a cardiomyocyte (CM); the dashed line boxed areas show the close contacts between Tps, Tps and CM. B) High-magnification TEM micrograph of the red dashed line boxed areas shown in (A) with the details of hetero-cellular close contacts (red arrow) between $\mathrm{Tp} 1$ and CM. C,D) High magnification TEM micrograph of the white and black dashed line boxed areas shown in (A) with details of homocellular close contacts (blue arrows) between Tps, respectively. mf, myofibrils; $\mathrm{m}$, mitochondrion; coll, collagen fibers; black arrowheads, caveolae.

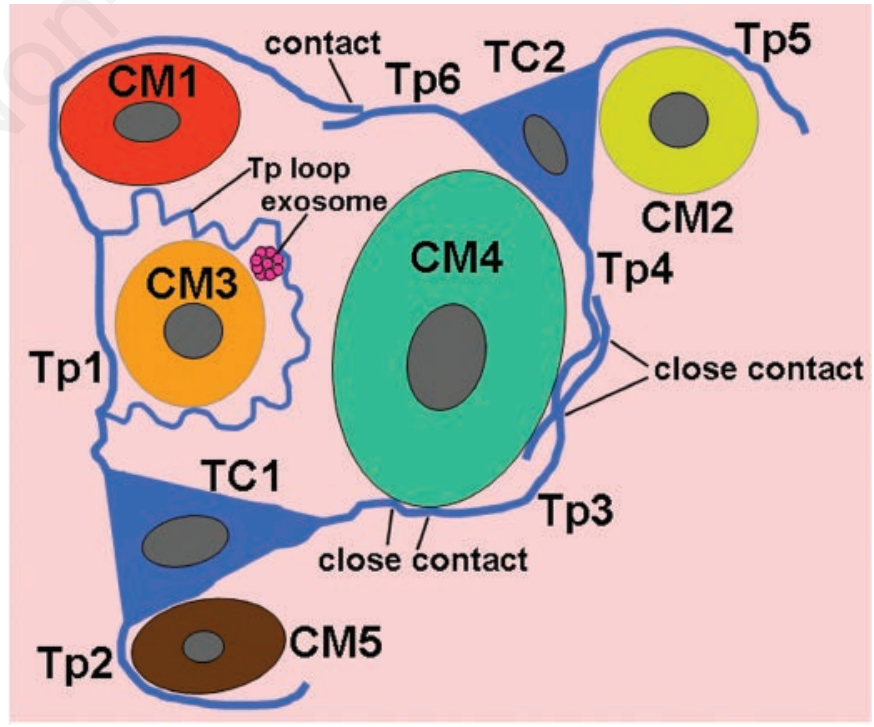

Figure 6. A schematic diagram of structural relationships between telocytes (TC) and cardiomyocytes (CM) in the myocardium of $A$. davidianus. $\mathrm{Tp}$, telopode. 
Tps form a closed tortuous loop and surround CM (Tp1 in Figure 4A), and they also form a non-closed tortuous loop (Tp3 in Figure 4A). The structural relationships between $\mathrm{TCs} / \mathrm{Tps}$ and surrounding $\mathrm{CM}$ are drawn in Figure 6.

\section{Discussion}

In the present study, by light microscopy and TEM, the cardiac TCs were observed in a lower amphibian, Andrias davidianus. The cardiac TCs of the $A$. davidianus possessed classical ultrastructural characteristics, including Tps with podoms and podomers, caveolae, dichotomous branchings, exosomes and close contacts with other Tps and surrounding CM. In lower vertebrates, including zebrafish and newt, cardiac TCs form a tandem with $\mathrm{CM}$, and play a key role in coordinating intercellular signaling, homeostasis maintenance and $\mathrm{CM}$ regeneration/repair. ${ }^{5,10,15}$ ${ }^{17}$ Previous studies showed that TCs and cardiac lymphatic endothelial cells have similar morphological traits, including long processes. ${ }^{18}$ The long Tps of TCs frequently establish structural relationships by nonclosed and discontinuous contacts in this study. However, lymphatic endothelial cells make up a lymphatic vessel and form a hollow, continuous and closed lumen to prevent lymph outleakage. In the present study, TCs formed a closed loop to surround tightly CM instead of lymph. Moreover, Tps of TCs have podoms and podomers, but cytoplasmic processes of lymphatic endothelial cells have no these structures. Therefore, TCs can be distinguished from lymphatic endothelial cells.

In the present study, TCs/Tps were frequently observed surrounding tightly $\mathrm{CM}$. Moreover, Tps established hetero-cellular close contacts with CM. TCs formed a network by their long Tps and homo-cellular close contacts between Tps. Previous studies demonstrated that Tps network plays a mechanical supporting role in cardiac interstitium. ${ }^{10,19}$ The exosomes were also found in vicinity of TCs. It is suggested that TCs might also communicate with $\mathrm{CM}$ via hetero-cellular close contacts and exosomes. ${ }^{20}$ Previous studies in newt have confirmed that a critical mechanism of the cardiac regeneration is that mature $\mathrm{CM}$ possesses the ability to trans- and re-differentiate into different cell types, by $\mathrm{CM}$ proliferation and subsequent cardiac regeneration after injury. ${ }^{21-23}$ Moreover, TCs play a role in process of cardiac regeneration and repair via mature $\mathrm{CM}$ proliferation in heart of amphibian. ${ }^{5,10,15,16,24}$ The cardiac TCs of the $A$. davidianus might regulate and induce $\mathrm{CM}$ to re-differentiate and trans-differentiate into different cell types, following division and proliferation. Accordingly, amphibian TCs might play regenerative and repair role in myocardium. Further studies are needed to disclose the differential roles of cardiac TCs, such as regulating stem/progenitor cells, and inducing mature cell types to regeneration. TCs are likely to become an up-and-coming source of cell therapy in cardiac regenerative medicine. . $^{825-27}$

This study confirmed the presence of cardiac TCs of an amphibian by TEM, and disclosed the ultrastructural relationships between TCs and CM. These results will improve the insight of the cytologic mechanism of heart regeneration via TCs-induced $\mathrm{CM}$ re-differentiation and proliferation.

\section{References}

1. Cantarero Carmona I, Luesma Bartolome MJ, Junquera Escribano C. Identification of telocytes in the lamina propria of rat duodenum: transmission electron microscopy. J Cell Mol Med 2011; 15:26-30.

2. Cretoiu D, Cretoiu SM, Simionescu AA, Popescu LM. Telocytes, a distinct type of cell among the stromal cells present in the lamina propria of jejunum. Histol Histopathol 2012;27: 1067-78.

3. Popescu LM, Faussone-Pellegrini MS. TELOCYTES - a case of serendipity: the winding way from Interstitial Cells of Cajal (ICC), via Interstitial CajalLike Cells (ICLC) to TELOCYTES. J Cell Mol Med 2010;14:729-40.

4. Shoshkes-Carmel M, Wang YJ, Wangen- steen KJ, Tóth B, Kondo A, Massasa EE, et al. Subepithelial telocytes are an important source of Wnts that supports intestinal crypts. Nature 2018;557:242-6.

5. Díaz-Flores L, Gutiérrez R, Díaz-Flores L Jr, Goméz MG, Sáez FJ, Madrid JF. Behaviour of telocytes during physiopathological activation. Semin Cell Dev Biol 2016;55:50-61.

6. Ibba-Manneschi L, Rosa I, Manetti M. Telocyte implications in human pathology: An overview. Semin Cell Dev Biol 2016;55:62-9.

7. Bei Y, Zhou Q, Sun Q, Xiao J. Telocytes in cardiac regeneration and repair. Semin Cell Dev Biol 2016;55:14-21.

8. Marini M, Rosa I, Ibba-Manneschi L, Manetti M. Telocytes in skeletal, cardiac and smooth muscle interstitium: morphological and functional aspects. Histol Histopathol 2018;33:1151-65.

9. Kucybala I, Janas P, Ciuk S, Cholopiak W, Klimek-Piotrowska W, Holda MK. A comprehensive guide to telocytes and their great potential in cardiovascular system. Bratisl Lek Listy 2017;118: 302-9.

10. Kostin S. Myocardial telocytes: a specific new cellular entity. J Cell Mol Med 2010;14:1917-21.

11. Pulze L, Baranzini N, Girardello R, Grimaldi A, Ibba-Manneschi L, Ottaviani E, et al. A new cellular type in invertebrates: first evidence of telocytes in leech Hirudo medicinalis. Sci Rep 2017;7:13580.

12. Zhang H, Zhong S, Ge T, Peng S, Yu P, Zhou $Z$, et al. Heterogeneous vesicles in mucous epithelial cells of posterior esophagus of Chinese giant salamander (Andrias davidianus). Eur J Histochem 2015;59:19-23.

13. Zhang H, Yu P, Zhong S, Ge T, Peng S, Zhou Z, et al. Gliocyte and synapse analyses in cerebral ganglia of the Chinese mitten crab, Eriocheir sinensis: ultrastructural study. Eur J Histochem 2016;60:2655.

14. Zhang H, Zhong S, Yu P, Ge T, Peng S, Guo X, et al. Telocytes in gastric lamina propria of the Chinese giant salamander, Andrias davidianus. Sci Rep 2016; 6:33554.

15. Popescu LM, Gherghiceanu M, Manole CG, Faussone-Pellegrini MS. Cardiac renewing: interstitial Cajal-like cells nurse cardiomyocyte progenitors in epicardialstem cell niches. J Cell Mol Med 2009; 13:866-86.

16. Gherghiceanu M, Popescu LM. Cardiac telocytes - their junctions and functional implications. Cell Tissue Res 2012; 348:265-79.

17. Milon A, Pawlicki P, Rak A, Mlyczynska E, Płachno BJ, Tworzydlo $\mathrm{W}$, et al. Telocytes are localized to testis of the bank vole (Myodes glareolus) and are affected by lighting conditions and G-coupled membrane estrogen receptor (GPER) signaling. Gen Comp Endocrinol 2019;271:39-48.

18. Rusu MC, Hostiuc S. Critical review: Cardiac telocytes vs cardiac lymphatic endothelial cells. Ann Anat 2019;222: 40-54.

19. Tay H, Vandecasteele T, Van den Broeck W. Identification of telocytes in the porcine heart. Anat Histol Embryol 2017;46:519-27.

20. Kostin S. Cardiac telocytes in normal and diseased hearts. Semin Cell Dev Biol 2016;55:22-30.

21. Laube F, Heister M, Scholz C, Borchardt T, Braun T. Re-programming of newt cardiomyocytes is induced by tissue regeneration. J Cell Sci 2006;119: 4719-29.

22. Bloomekatz J, Galvez-Santisteban M, Chi NC. Myocardial plasticity: cardiac 
development, regeneration and disease. Curr Opin Genet Dev 2016;40:120-30.

23. Borchardt T, Braun T. Cardiovascular regeneration in non-mammalian model systems: what are the differences between newts and man? Thromb Haemost 2007;98:311-8.

24. van Amerongen MJ, Engel FB. Features of cardiomyocyte proliferation and its potential for cardiac regeneration. J Cell Mol Med 2008;12:2233-44.

25. Popescu LM, Fertig ET, Gherghiceanu M. Reaching out: junctions between cardiac telocytes and cardiac stem cells in culture. J Cell Mol Med 2016;20: 370-80.

26. Yang $\mathrm{P}$, Ahmed N, Ullah S, Chen Q, Zheng Y. Features of telocytes in agricultural animals. Adv Exp Med Biol 2016;913:105-13.

27. Marini M, Mencucci R, Rosa I, Favuzza E, Guasti D, Ibba-Manneschi L, et al. Telocytes in normal and keratoconic human cornea: an immunohistochemical and transmission electron microscopy study. J Cell Mol Med 2017;21:3602-11. 